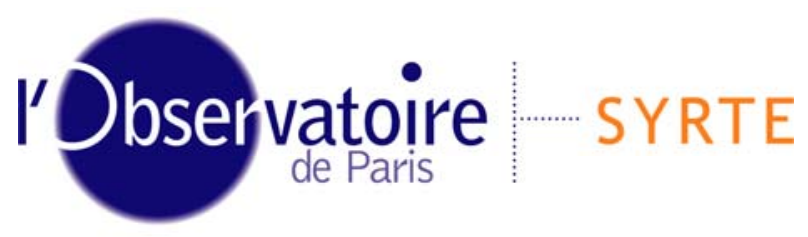

Systèmes de Référence Temps-Espace

\title{
Ultra-Stable Ground Frequency Dissemination via Optical Fibres*
}

\author{
F. Narbonneau, M. Lours, O. Lopez*, G. Santarelli. \\ LNE-SYRTE, Observatoire de Paris, \\ 61, avenue de l'Observatoire \\ F-75014 Paris, France \\ *Laboratoire de Physique de Lasers, Univ. Paris XIII, Villetaneuse, France.
}

Abstract

\begin{abstract}
In this report we present a few results on a dissemination system of ultra-stable reference signals at $100 \mathrm{MHz}$ and $1 \mathrm{GHz}$ on a standard fibre network. The signals are simply transferred by amplitude modulation of an optical carrier. Two different approaches for compensating the noise introduced by the link have been implemented. The limits of the two systems are analyzed and several solutions suggested in order to improve the frequency stability and to further extend the distribution distance. Nevertheless, our system is a good tool for the best cold atom fountains comparisons between laboratories, up to $100 \mathrm{~km}$, with a relative frequency resolution of $10^{-14}\left(3-5 \times 10^{-15}\right)$ at one second integration time and $10^{-17}\left(2-3 \times 10^{-18}\right)$ for one day of measurement for a $100 \mathrm{MHz}$ reference signal $(1 \mathrm{GHz})$. A further extension of the link using fiber spools and optical amplifiers over $186 \mathrm{~km}$ is also reported.
\end{abstract}

I Introduction: Scientific and technical objectives

\section{Principle and objective}

III Active phase fluctuations compensation systems

IV Characterization of the two different 100MHz systems in a full bi-directional link of $43 \mathrm{~km}$

\section{Upgraded Compensators at 1GHz}

VI A Pan European network

\author{
VII Conclusions
}

Appendix

Acknowledgments

References

\footnotetext{
${ }^{*}$ ESA Contract $n^{\circ} 17367 / 03 / \mathrm{D} / \mathrm{SW}(\mathrm{SC})$
} 


\section{Introduction: scientific and technical objectives}

Ultra-stable frequency and time sources play an important role in many modern Time and Frequency metrology and fundamental physics applications (clock evaluation, relativity tests, fundamental constants test ...)(e.g. [1-4]). In the field of particles physics, modern large linear accelerators require RF distribution system with minimal phase drifts and errors for the neutrons and positrons generation [5]. In radio-astronomy, e.g. in the case of the ALMA (Atacama Large Millimetric Array) project or for VLBI (Very Long Baseline Interferometry), the combination of high frequency and long baselines of the interferometer needs the distribution of a local oscillator with low phase noise and low phase drift through the array [6,7]. In the case of Deep Space Networks, atomic clock reference signals need to be distributed to all frequency users with as little degradation as possible. In some cases, these users are far away from the location of the reference, which imposes the use of optical fibre links [8,9]. Modern cold atoms frequency standards in the microwave domain have already demonstrated accuracy in the $10^{-15}$ range with the potential to reach the $10^{-16}$ level or better. Frequency stabilities, defined by the Allan standard Deviation (ADEV, see Appendix), are commonly of $10^{-13} \tau^{-1 / 2}$ for such standards and a few $10^{-14} \cdot \tau^{-1 / 2}$ have been demonstrated using more advanced techniques [10]. Cold atom optical clocks have the potential to reach the $10^{-17}$ accuracy level [11-14]. The emergence of modern microwave-tooptical synthesizers based on mode-locked femtosecond lasers allows high resolution comparisons between microwave and optical clocks [15-17]. Clocks comparisons are currently performed by satellite, as for example GPS or TWSTFT (Two-Way Satellite Time and Frequency Transfer. Measurements are limited by the transmission system to about $10^{-15}$ at one day averaging time [18]. These methods are thus insufficient for measuring the ultimate performance of a microwave or an optical standard (Fig.1). Upgrades of the orbital equipments are expectable to improve the current performance, but are quite complex and expensive. Moreover, the two previous systems deliver only a synchronization signal not allowing direct short-term stability comparisons.

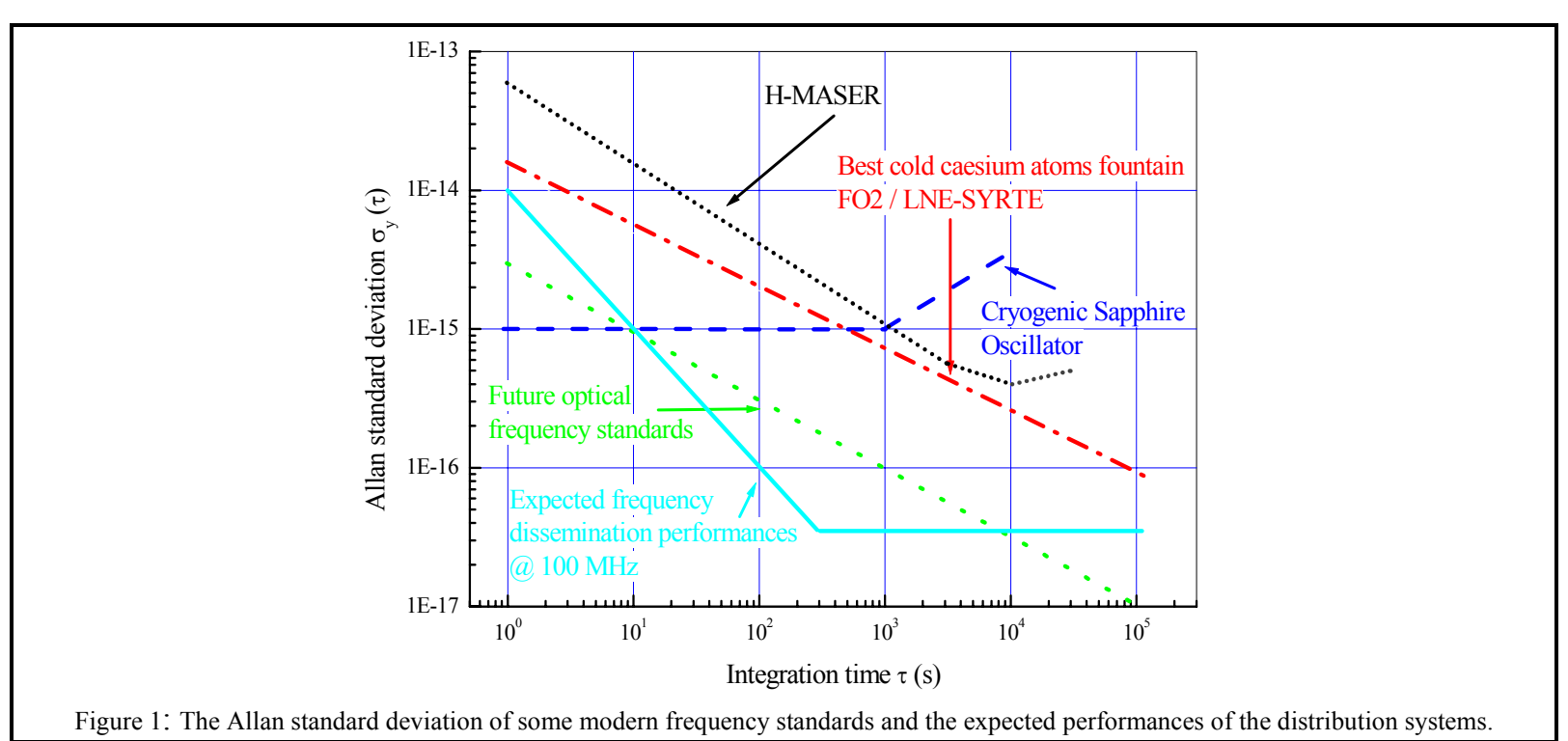

Then for much of applications a reference signal is needed. Hence, the opportunity to compare microwave and optical clocks by the development of a new type of frequency dissemination by optical fibre seems appropriate, even when the laboratories are separated by $100 \mathrm{~km}$ or more [19-21].One can indeed take advantage of both the low attenuation and low dispersion in the fibre, which allows reaching long distance frequency transfer by maintaining a good signal-to-noise ratio (SNR). Moreover the access to an ultra-stable frequency reference for a large number of laboratories opens the way to perform new experiments in fundamental physics. The development and operation of a state-of-the-art frequency standard remain a strong limitation and can be overcome by a fibre distribution system connecting Time and Frequency Metrology laboratories to users. The simplest way to develop a fibre distribution is to use the redundancy of the telecom network. In this paper, we present the transfer of high frequency stability signal at $100 \mathrm{MHz}$, by using the existing telecommunication fibre network, over a few tens kilometres, with compensation of the phase noise introduced by the link.

\section{Principle and objective.}

The goal of the dissemination is the distribution of a reference signal at a frequency of $100 \mathrm{MHz}$ or $1 \mathrm{GHz}$, synthesized from a frequency standard, by amplitude modulation of an optical carrier, without degradation of the phase noise of the distributed signal. The reference signal modulates the bias current of a DFB laser diode, at $1.55 \mu \mathrm{m}$, which is transmitted through a fibre optical link to users. At the link extremity, a photodiode detects the amplitude modulation and converts the optical signal to an electrical signal oscillating at the reference frequency and phase coherent with the microwave reference source. The high stability and low 
phase noise of the transferred signal are degraded by the residual phase noise of the optical link and by the attenuation in the fibre. We operate in urban environment by using the existing telecom network. Thus, fibre layout and installation aspects are not ideal and the stability of the optical link can be affected by environmental effects. Optical length of the fibre is modified by mechanical stresses and temperature fluctuations. The first one affects phase noise and short-term frequency stability performances of the transmitted signal. The second effect is a slowly changing phenomenon and has an impact on the long-term stability. These instabilities have been studied on two optical links showed in figure 2, using the dense France Telecom network and connecting LNE-SYRTE to Laboratoire de Physique des Lasers (LPL) (about $43 \mathrm{~km}$ ), and LNE-SYRTE with Laboratoire Kastler Brossel (LKB-University Paris VI) (about $3 \mathrm{~km}$ ). Measurements, realized at different periods, are presented in figure 2 and show nonstationary effects depending on the activities around the link. Periodic effects as daily temperature variations appear as a bump at the half period, on the ADEV.

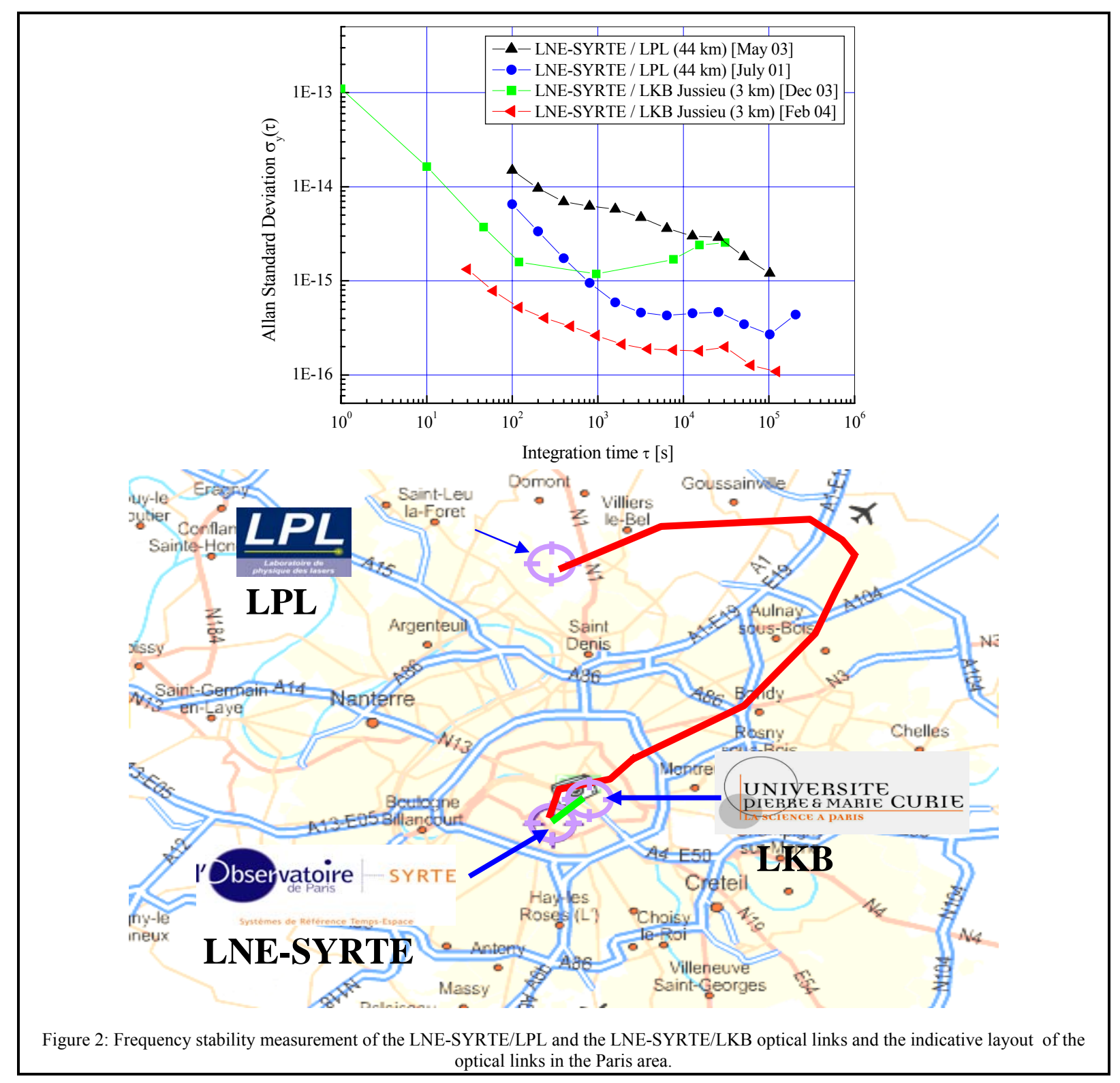

For example, if we consider a sinusoidal perturbation of $0.2^{\circ} \mathrm{C}$ with a period of $1000 \mathrm{~s}$ due to air conditioning and acting on a section of 50 meters of the optical link, the ADEV of the link could be limited to about $7 \times 10^{-16}$ at $500 \mathrm{~s}$ integration time. In the same way, a daily $0.5^{\circ} \mathrm{C}$ temperature variation on 43 kilometres of optical fibre is converted into instability of the order of $1.3 \times 10^{-}$ ${ }^{14}$ at $43200 \mathrm{~s}$ averaging time. Consequently, the distribution system needs an active control loop to compensate for these phase variations induced on the signal transmitted through the link related to the environment (mechanical vibrations, temperature fluctuations ...). 
The objective of the dissemination being clock comparisons or delivery of a reference signal coming from an H-Maser or a Cryogenic Sapphire Oscillator (CSO), the compensation set-up must introduce a phase noise lower than the reference signal. In this perspective we have to develop a system which delivers a reference signal at $100 \mathrm{MHz}(1 \mathrm{GHz})$, showing a relative frequency stability $\sigma_{\mathrm{y}}(\tau) \sim 2 \times 10^{-14}\left(2 \times 10^{-15}\right)[\tau=1 \mathrm{~s}]\left(<10^{-16}\left(<10^{-17}\right) @ 1 \mathrm{~d}\right)$, that implies a residual flicker phase noise of $-120 \mathrm{dBrad} / \mathrm{Hz}(-$ $130 \mathrm{dBrad}^{2} / \mathrm{Hz}$ ) at $1 \mathrm{~Hz}$ and a white phase noise floor with a level of $-140 \mathrm{dBrad}^{2} / \mathrm{Hz}$ for the $100 \mathrm{MHz}$ and $1 \mathrm{GHz}$ respectively.

\section{Active phase fluctuations compensation systems}

\section{A Presentation.}

The principle of the phase fluctuations compensation is displayed in figure 3. At the link extremity, the detected signal can not be directly compared to the reference signal and thus the correction of the phase perturbations can be only carried out at the link emission. A two-way distribution, using the same optical fibre link, allows determination of the phase perturbation accumulated along a full round trip with the hypothesis that the forward and the backward signals are corrupted by the same perturbation. The compensation rests then on the measurement of the phase of the signal after one round trip to apply a correction on the emitted signal. The reference signal at the frequency $f_{\text {ref }}=\omega_{\text {ref }} / 2 \pi$ is used for modulating a laser diode. The amplitude modulated signal is then corrected by a phase term $\phi_{c}$ This correction term is provided either by phase shifting the RF modulating signal or by modifying the propagation delay in the fibre. At the user-end, the signal corrupted by the environmental perturbations is detected:

$$
\mathrm{V}_{\mathrm{rf} \text { detected }}(t) \propto \sin \left(\omega_{r e f} t+\phi_{\text {ref }}+\phi_{p}+\phi_{c}\right)
$$

This signal is split in two signals: one part for the user applications and the other to be re-injected via an optical circulator in the same optical fibre. After one round-trip, the signal, twice corrupted by the term $\phi_{p}$ is detected. A RF process allows generation of an error signal, applied to the phase corrector. Two different laser sources, operating at slightly different wavelengths, are used for generating the forward and the backward optical signals and optical add/drop functions are realized with optical circulators. Different approaches of phase compensation have been studied and are described here.

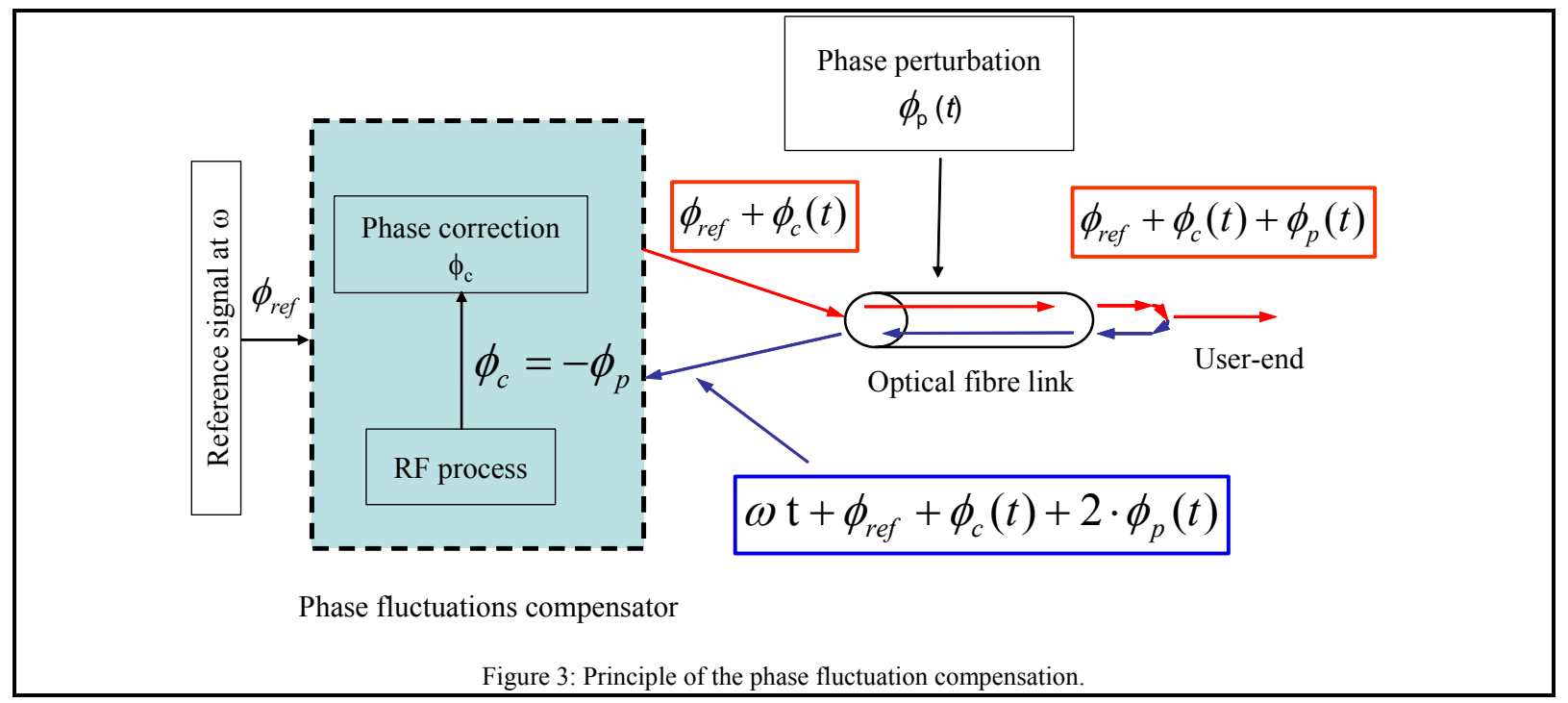

\section{B. Electronic phase fluctuations compensator}

In the case of an electronic phase fluctuations compensator (fig. 3), the correction is performed by acting on the phase of the injected signal in the optical link, that we call $\phi_{\text {input }}$. We define by $\phi_{r}$ the phase of the round-trip signal, and $\phi_{\text {output }}$ the phase | of the detected signal at the_user-end, equal to:

$$
\phi(t)=\phi(t-\tau)+\int_{t-\tau}^{t} \phi_{p}(\xi) d \xi
$$


where $\tau$ is the propagation delay in the optical fibre link and $\phi_{p}(x)$ is the distributed phase perturbation along the fibre.

The main effect of the delay $\tau$ is to limit the loop bandwidth. In the following discussion, we neglect the influence of the delay. The output signal must be phase coherent with the reference source of frequency $\omega_{\text {ref }}$ and on average of phase $\phi_{\text {ref }}$, and thus the correction applied to the emitted signal must be equal to the opposite of the phase perturbation $\phi_{p}$ Consequently, on average (or for time much longer than $\tau$ ) the phase of the input signal, $\phi_{\text {input }}$ is:

Then, the phase of the round-trip signal becomes:

$$
\phi_{\text {input }}=\phi_{\text {ref }}-\phi_{p}
$$

$$
\begin{aligned}
\phi_{r} & =\phi_{\text {input }}+2 \phi_{p} \\
& =\phi_{\text {ref }}+\phi_{p}
\end{aligned}
$$

The phase coherence of the output signal is hence imposed by maintaining a conjugation relationship between the input and the round trip signal of the optical link:

$$
\left(\phi_{\text {input }}-\phi_{\text {ref }}\right)=-\left(\phi_{p}-\phi_{r}\right)
$$

A simplified scheme of the phase conjugator is shown in figure Figure 4. The correction is performed with a phase shifter in series with the reference signal, which is used as the input signal. The reference signal is power divided to drive two phase detectors. Phase detection between the reference signal, the input corrected signal and the round-trip signal, allow generation of two baseband signals, connected to the inputs of a low noise differential amplifier. The output of this amplifier is used for driving a loop filter, controlling the electronic phase shifter until the phase conjugation, and thus a zero level at the amplifier output is reached. In spite of the simplicity of operation, this system suffers from various drawbacks.

First, the phase correction is limited by the dynamic of the phase shifter. Electronic phase shifters have a typical dynamic of 180 degrees with a non linear response, inducing variable insertion losses. Moreover the phase shifter can present a phase noise excess, compared to the other components of the phase conjugator. Secondly, phase detectors are quite sensitive to the driving levels and it is difficult to ensure the same sensitivity for the two detectors of figure 4 . The practical realization leads to a very poor effective system of the phase perturbations cancellation.

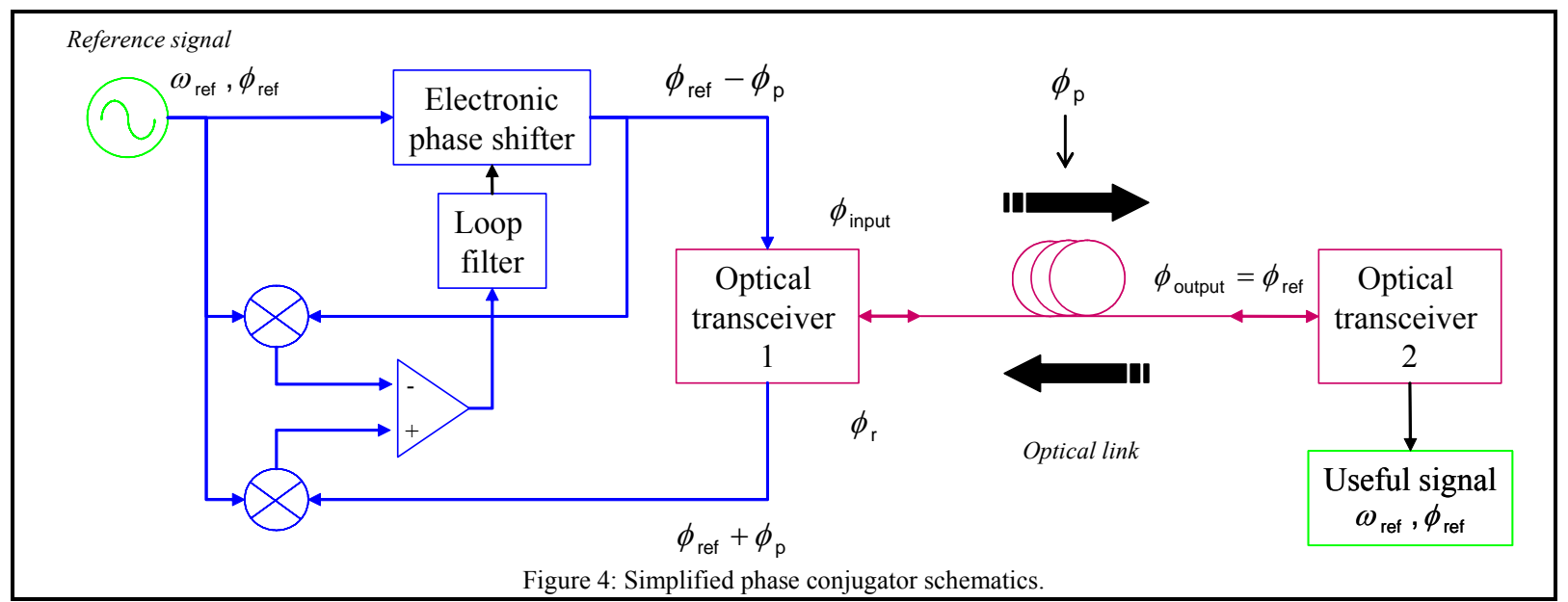

A new scheme, regarding the previous considerations and introduced by the JPL [22] is shown in figure Figure 5. Two symmetrical signals are produced by frequency shift $f_{\text {shift }}$ of the reference signal $f^{ \pm}=f_{\text {ref }} \pm f_{\text {shift }}$. This scheme allows replacement of the double phase measurements (Fig4) by a much more accurate double frequency mixing and a single phase measurement. 


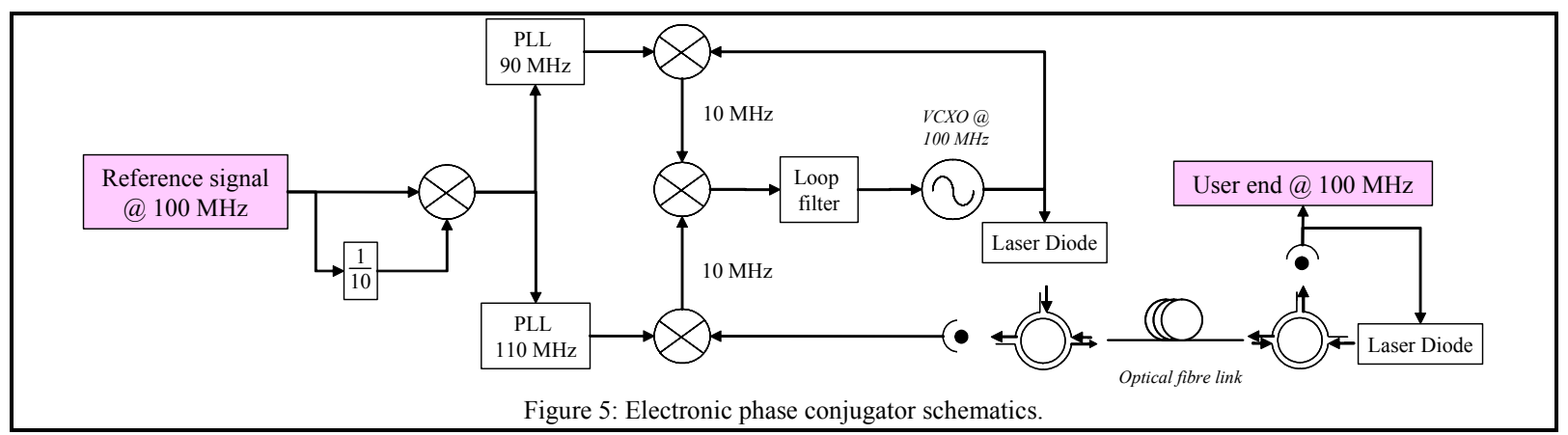

The dynamic and the linearity of the phase correction is improved by using a voltage controlled quartz oscillator (VCXO), as a phase shifter, delivering a signal at the reference frequency with a stable amplitude. The VCXO presents thus the advantage to correct all phase perturbation in the correction bandwidth of the phase compensator, which is limited by the round-trip propagation delay in the optical link (about $0.3 \mathrm{~ms}$ in the case of the 43-km LNE-SYRTE to LPL optical link). The $100 \mathrm{MHz}$ output signal of the VCXO modulates the bias current of the DFB laser diode. The optical signal is launched in the optical fibre link to the user. At the user end, a simple system allows detection and regeneration of the backward signal. The detected signal after a one-way distribution is proportional to:

$$
V_{\text {user end }}(t) \propto \sin \left(\omega_{\text {osc }} t+\phi_{\text {osc }}+\phi_{p}\right)
$$

The backward optical signal is submitted to the same phase perturbation and after one complete round-trip, the detected signal has the following form:

$$
V_{\text {round trip }}(t) \propto \sin \left(\omega_{\text {osc }} t+\phi_{\text {osc }}+2 \phi_{p}\right)
$$

The servo loop forces the VCXO at $100 \mathrm{MHz}$ both to be phase coherent with the reference source and to compensate for the phase perturbation. For obtaining the phase conjugation, two signals separated by $10 \mathrm{MHz}$ around the reference frequency (one at 90 $\mathrm{MHz}$ and the other at $110 \mathrm{MHz}$ ) are produced by frequency mixing between the reference signal and itself frequency divided by ten. Two different systems, based on PLL (Phase Lock Loop) are used for filtering each signal issue from the previous frequency mixing. The signal, from the "down conversion", at $90 \mathrm{MHz}$, is mixed with the modulating signal, delivered by the VCXO, to obtain a signal at $10 \mathrm{MHz}$ :

$$
V_{1}(t) \propto \sin \left[\left(\omega_{o s c}-2 \pi x 90 M H z\right) t+\phi_{o s c}-\frac{9}{10} \phi_{r e f}\right]
$$

In parallel, the signal at $110 \mathrm{MHz}$ is mixed with the round-trip signal, producing another signal at $10 \mathrm{MHz}$ :

$$
V_{1}(t) \propto \sin \left[\left(2 \pi x 110 M H z-\omega_{o s c}\right) t-\phi_{o s c}+\frac{11}{10} \phi_{r e f}-2 \phi_{p}\right]
$$

The phase comparison at $10 \mathrm{MHz}$ allows generation of a base-band signal, containing the three phase terms:

$$
V_{\text {error }}(t) \propto \phi_{\text {osc }}+-\phi_{\text {ref }}+\phi_{p}
$$

which is cancelled in normal operation. The phase of the VCXO is then:

$$
\phi_{\text {osc }}=\phi_{\text {ref }}-\phi_{p}
$$

By this process, the stability and the accuracy of the reference source is transmitted to the user end in the system bandwidth. The capacity of the phase compensator to reject phase perturbations in the control bandwidth is defined by the rejection factor, equal to the ratio between the phase variations in open and in closed loop. The performance of the distribution system depends both of the intrinsic system phase noise and of the rejection factor.

A dedicated set-up is used for the characterization of the phase conjugator. Simulation of phase perturbations are realized by periodically heating a $2.5 \mathrm{~km}$ fibre spool with amplitude of $4^{\circ} \mathrm{C}$ and a period of about $4000 \mathrm{~s}$. This perturbation induces a phase modulation of the order of $200 \mathrm{mrad}$ on the $100 \mathrm{MHz}$ transmitted signal. In operation, when the phase conjugator is activated, the 
residual phase modulation measured at the link output is reduced to $0.4 \mathrm{mrad}$, that implies a rejection factor of the phase perturbations along the link of about $500(53 \mathrm{~dB})$. Moreover, the complete distribution system including the phase conjugator shows a flicker phase noise with a level of $-123 \mathrm{dBrad}^{2}$ at $1 \mathrm{~Hz}$ and a white noise floor below $-140 \mathrm{dBrad} / \mathrm{Hz}$. This ensures the possibility to transfer metrological signal with a frequency stability $\sigma_{\mathrm{y}}(\tau)$ below than $1 \mathrm{x}^{-14}$ at 1 second averaging time. This system was implemented at SYRTE.

\section{Optical compensation system}

An optoelectronic compensation system has also been developed and implemented at LPL, operating in a slightly different way. The phase correction is applied both on the emitted and on the backward signal by directly acting on a section of optical fibre, placed in series with the optical link. The phase correction is then performed by modifying the optical propagation delay (and thus the optical path) of the optical signal in the fibre link.

The principle of the optoelectronic phase compensation is presented in figure 6. For increasing isolation between the two ways of the distribution system, two different modulation frequencies are used: $1 \mathrm{GHz}$ for the emission and $100 \mathrm{MHz}$ for the return way. Consequently, a $1 \mathrm{GHz}$ signal must be generated from the reference $100 \mathrm{MHz}$ signal by a frequency synthesizer. The optical signal is distributed in the fibre link through the optical compensator and thus corrected. At the user-end, the detected signal has the following form:

$$
V_{\text {user }}(t) \propto \sin \left[(2 \pi x 1 G H z) t+10 \phi_{\text {ref }}+\phi_{\text {correction }}^{\prime}+\phi_{p}^{\prime}\right\rfloor
$$

where $\phi_{\text {correction }}^{\prime}, \phi_{p}^{\prime}$ are respectively the correction term and the perturbation applied to the transmitted signal at $1 \mathrm{GHz}$. A second frequency synthesis chain allows delivering a signal at $100 \mathrm{MHz}$ from the $1 \mathrm{GHz}$ detected signal. This signal is used for modulating a second laser diode to produce the backward optical signal. This signal is corrupted by the same perturbation and also corrected. After one round trip, we detect a $100 \mathrm{MHz}$ signal proportional to:

$$
V_{\text {user }}(t) \propto \sin \left[(2 \pi x 100 M H z) t+\phi_{\text {ref }}+2\left(\phi_{\text {correction }}^{\prime}+\phi_{p}^{\prime}\right)\right]
$$

with:

$$
\phi_{\text {correction }}=\frac{\phi_{\text {correction }}^{\prime}}{10}, \quad \phi_{p}=\frac{\phi_{p}^{\prime}}{10}
$$

The phase comparison between the reference signal at $100 \mathrm{MHz}$ and the round-trip signal leads to generation of a baseband error signal:

$$
V_{\text {error }}(t) \propto \phi_{\text {correction }}+\phi_{p}
$$

which is applied to a loop filter to drive the optical phase corrector. This corrector is composed of two sub-systems. Fast and small phase fluctuations (mechanical vibrations, fast temperature variations induced by air conditioning eg.) are corrected by changing the length of a portion of optical fibre with a piezo-electric actuator.

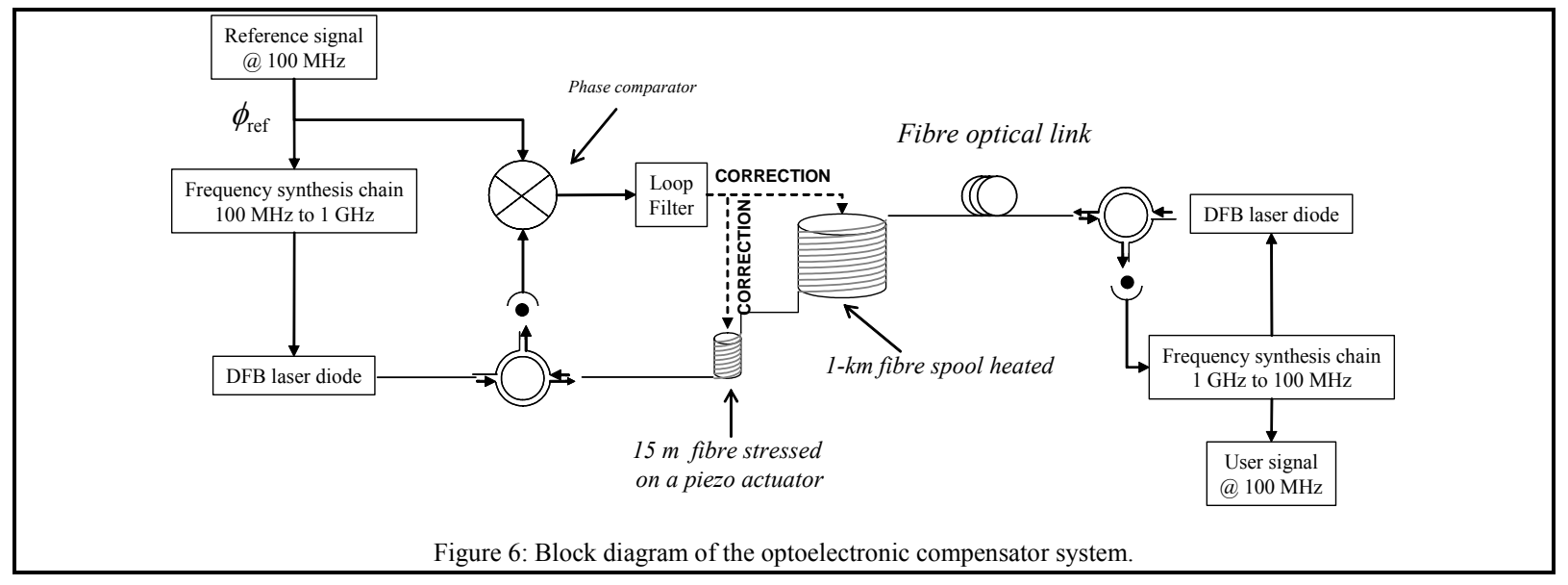

A 15 -meter optical fibre is wrapped around a 5 -cm diameter PZT of about $10 \mu \mathrm{m}$ variation under $1 \mathrm{kV}$ voltage, and is mechanically stretched, allowing correction up to about $15 \mathrm{ps}$ or $10 \mathrm{mrad}$ at $100 \mathrm{MHz}$, in a bandwidth of a few hundreds $\mathrm{Hz}$. Slow and large perturbation are compensated by heating a $1-\mathrm{km}$ fibre spool introduced along the link $\left(40 \mathrm{ps} /{ }^{\circ} \mathrm{C}\right.$ or $25 \mathrm{mrad} /{ }^{\circ} \mathrm{C}$ at 100 $\mathrm{MHz}$ ). For the laboratory tests, a fibre stretcher and a heating system are placed along a laboratory link of a few kilometers to generate fast and slow phase perturbations. By this way, a temperature step of $10 \mathrm{C}$ is realized on a 1-km fibre spool, and the 
phase shift induced on the detected signal and measured in open and closed loop. Rejection factor of about 750 for slow phase perturbations is shown. In contrast, only 20-25 dB attenuation are observed on intentionally produced small and fast perturbations. The gain of the correction is limited by parasitic phase shifts generated by Polarization Dependent Losses (PDL) under mechanical stress of the fibre. The mechanical stress affects the geometry of the fibre which becomes birefringent. Thus the polarization of the transmitted beam changes and leads to an amplitude modulation (AM) of the detected signal on the tilted photodiode. AM is directly converted into PM (Phase modulation) and detected as a phase perturbation term which is compensated by the phase compensator. Corrections of phase perturbations are corrupted by this parasitic phenomena and the performance of the system is then degraded. PDL is only related to the optical signal and independent of the modulation frequency. By upgrading the system to higher operation modulation frequencies, this problem could be reduced proportionally to the frequency.

\section{Characterization of the two different 100MHz systems in a full bi directional link of $43 \mathbf{~ k m}$}

A full bidirectional compensated optical link $(2 \times 43 \mathrm{~km})$ has been achieved by using the two fibres of the LNE-SYRTE to LPL link and by implementing the two previous compensation systems. This link is composed of various sections of buried optical cables of the France Telecom metropolitan network. The continuity of each optical fibre of $43 \mathrm{~km}$ is ensured by optical splicing and a global attenuation of $12 \mathrm{~dB}$ on each fibre is measured. The low phase noise $100 \mathrm{MHz}$ local oscillator of LNE-SYRTE is transferred to LPL by using one of the two fibres of the link, and is phase compensated by the phase conjugator. At LPL, a signal phase coherent with the LNE-SYRTE local oscillator is detected and used as the input reference signal for the second optical link, connecting back LPL to LNE-SYRTE via the second $43 \mathrm{~km}$ fibre. The optoelectronic system is installed on this link to compensate for the phase perturbations. At LNE-SYRTE, we compare the phase detected signal coming from LPL with the local oscillator for characterizing the two dissemination systems. The use of two different systems allows systematic studies related to one of the two compensators, and thus allows having a full characterization of the distribution system. Figure 6 shows the residual frequency stability measurements of the full bidirectional link $(2 \times 43 \mathrm{~km})$ between LNE-SYRTE and LPL. An Allan standard deviation of $10^{-17}$ at one day and $1.2 \times 10^{-17}$ at one second averaging time is obtained for the frequency instabilities added by the compensated optical link. The ADEV is calculated from the measured phase data, low-pass filtered $(3 \mathrm{~Hz})$.

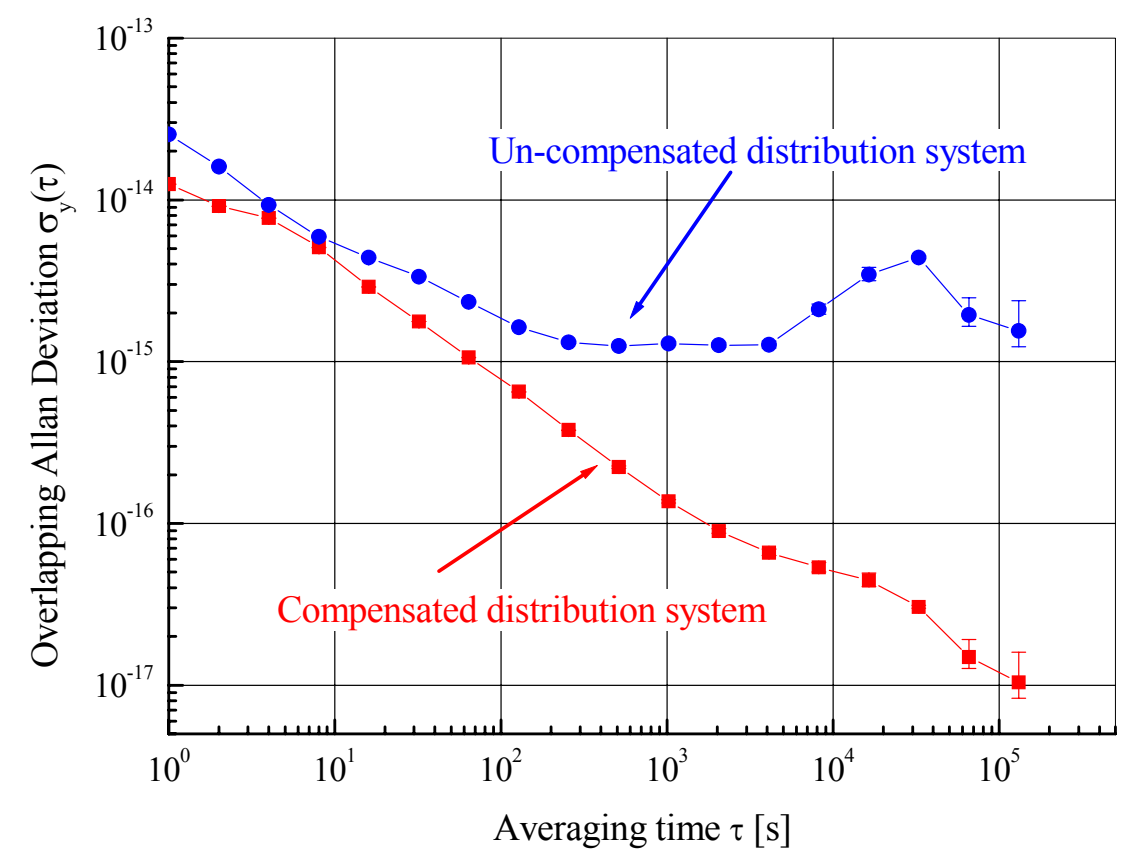

Figure 7: The Allan standard deviation of some frequency standards and the expected performances of the distribution system.

As shown in figure 7, the frequency stability in closed loop is better than the open loop for all integration times, between $1 \mathrm{~s}$ and 1 day. The short-term stability is mainly limited by the SNR at the detection, degraded by three contributions: the intrinsic noise of the distribution system, the optical attenuation in the fibre and the stimulated Brillouin scattering (SBS). Optical losses can be overcome by injecting powerful signals in the fibre, up to the Brillouin generation in order to avoid any laser source depletion. The direct amplitude modulation of the DFB bias current induces a broadening of the optical spectrum with a distribution of the energy within this spectrum and thus allows increasing the injected power level. The SBS leads also to a scattered wave detected as a white phase noise excess around the RF carrier with a few tens $\mathrm{MHz}$ width. This can be easily reduced by additional fibre Bragg grating filters (FBG), used in detection. The optoelectronic phase compensator, operating with two different modulation 
frequencies, separated by more than the Brillouin bandwidth, is less sensitive to this phenomenon. In spite of its complexity, the phase conjugator presents a better phase noise ensuring thus a better short-term performance.

The noise of the optoelectronic system is mainly degraded by additive amplitude noise, generated by the PZT corrector. One solution for improving the phase noise of each dissemination system is then to move to a higher operation RF frequency as 1 GHz. The long-term frequency stability is mainly limited by the phase conjugator which presents a rejection factor of only a few hundreds. Two main phenomena degrade the performance of the phase conjugator. Coherent leakage signals of $90 \mathrm{MHz}$ and 110 $\mathrm{MHz}$ may induce parasitic phase shifts. The second limitation comes from optical feedback due to reflections of optical connectors or optical splicing along the link. To ensure a sufficient compensation of the phase fluctuations introduced by the link, all parasitic noises should be $60 \mathrm{~dB}$ under the the detected signal. Such level could be reached by shifting the modulation frequency of the backward signal or by adding optical filters. Finally, we are also confronted by a polarization effect, PMD (Polarization Mode Dispersion), which are detected as a chromatic dispersion with a random coefficient, leading to a random propagation delay on each way of the dissemination. Due to PMD, the principle of the compensation, based on the measurement of twice the perturbation after one round trip is not valid any more. One solution is the polarization scrambling of the injected optical signal, faster than the loop bandwidth as presented in the next chapter.

\section{Upgraded Compensators at $1 \mathrm{GHz}$}

Both compensator electronic and optoelectronic have been upgraded to operate at $1 \mathrm{GHz}$ (Figures $8 \& 9$ ). The long-term frequency stability of the electronic compensator is limited to about $10^{-16}$ by stray optical reflections. Moreover, the signal-to-noise ratio is degraded by Brillouin back-scattering (SBS). To overcome these limitations, optical filters based on fibre Bragg grating have been added to the system to reduce by $30-40 \mathrm{~dB}$, all parasitic terms..

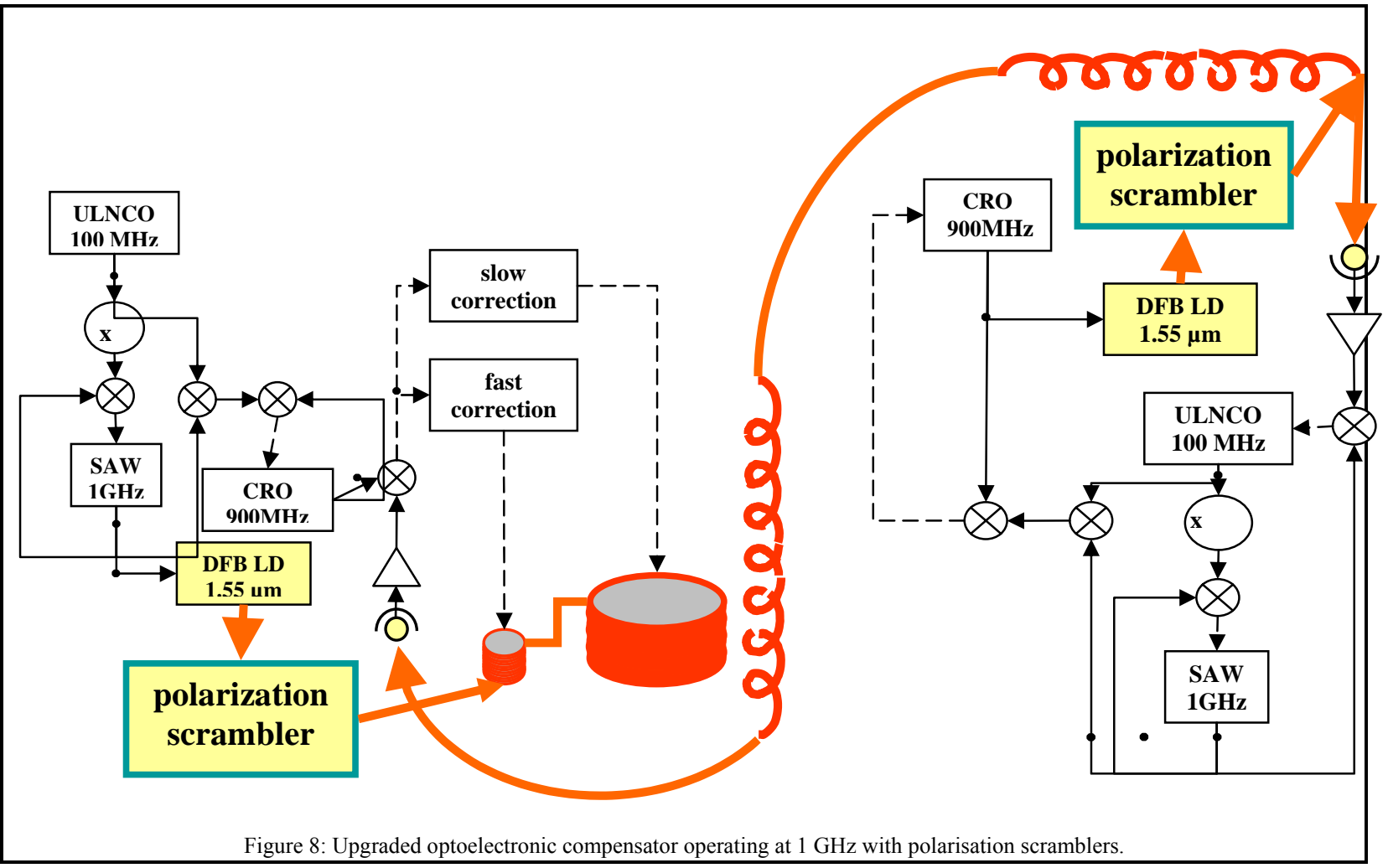

The limiting effect for long term stability of both compensators is the PMD induced by the birefringence of the optical fiber due to asymmetry of the core This PMD varies in time when the asymmetry is due to mechanical stress induced by vibrations or by temperature variations. The PMD limits also the short term stability of the optoelectronic compensator: the piezoelectrical fiber stretcher varies the polarization state of the transmitted signal and thus introduces a variable propagation delay which adds to the primary delay of the link. 


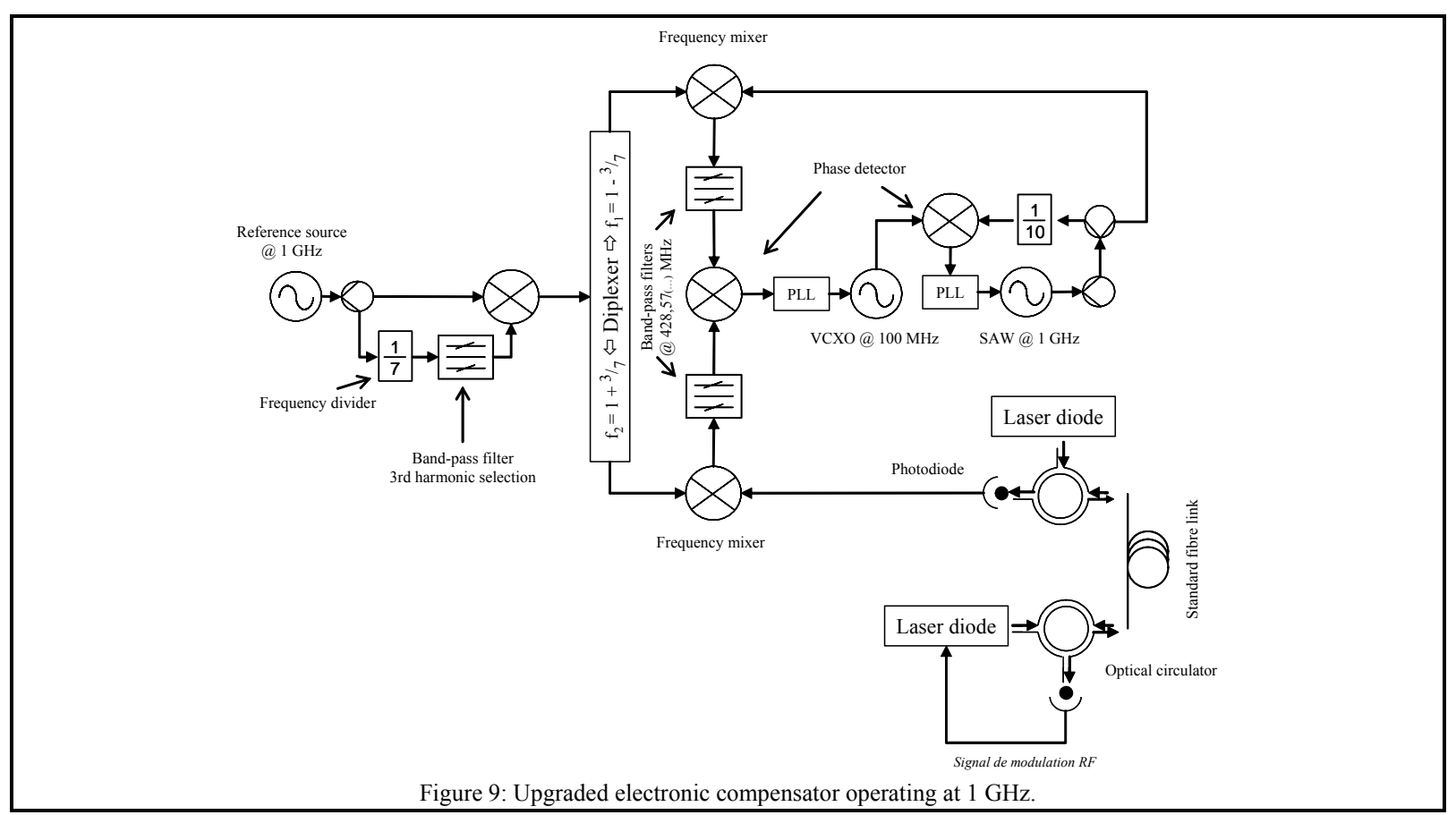

The slow variation in time of the Differential Group Delay (DGD) and direction axis of polarization of the fibre causes a random variation of the propagation delay not of same way for the propagation of forward signal and the back signal. The compensator floor is limited to a few $10^{-17}$ between $10^{4}$ and $10^{5}$ seconds integration time. The solution is to scramble the polarization of the injected signal at the emission with a frequency higher than the cut off frequency loop. In this case the DGD is averaged and the effects of PMD are minimized. For that purpose we use polarization scramblers (General Photonics) which have three axes acting in a same way as a variable retardation wave plate. Each axis is excited at different resonant frequency $(\sim 60, \sim 100, \sim 130 \mathrm{kHz})$ which allows exploring all polarization states. Figure 10 shows the ADEV which is calculated from the phase data measured on the compensated link and filtered with low-pass filter of $3 \mathrm{~Hz}$. We obtain residual frequency stabilities of the link of $5 \times 10^{-15}$ at 1 second integration time and $2 \times 10^{-18}$ at one day integration time for the optoelectronic compensator. This is the best stability ever achieved for an optical link over such a distance.

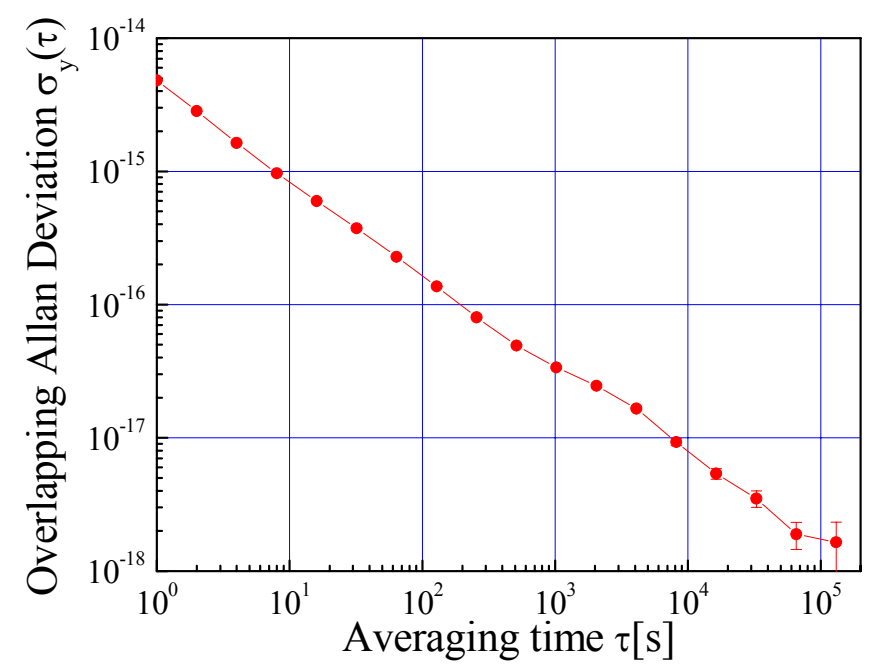

Figure 10: The Allan standard of the transferred signal over $86 \mathrm{~km}$. 
We have explored the possibility of increasing the link length to186 km by adding $100 \mathrm{~km}$ of fiber spools. Two Erbium Doped Fiber Amplifiers (EDFA) were added at half distance to compensate the $20 \mathrm{~dB}$ additional optical losses.

We obtain a rather good result on long term frequency stability, better than $10^{-17}$ at one day integration time while the short term is degraded by the EDFA excess noise. Figure 11 shows the preliminary measurement of the relative frequency stability of 186 $\mathrm{km}$ link for the optical compensator and for an electronic compensator. The two systems give about the same results.

\section{A Pan-European Network}

The obtained results which are summarized in Table 1 opens the way to establish a Pan European fiber network based on a sequence of compensated segments of 100-200 km. Existing academic of scientific well operational networks can be used as backbones for those links. The target stability for a $1000 \mathrm{~km}$ link falls in the low $10^{-17}$ for one day integration, about two orders of magnitude better than existing satellite based time and frequency transfer systems. The use of this network will range from T\&F metrology to fundamental Physics applications to the test of more advanced space based T\&F transfer systems and in VLBI.

The cost evaluation is difficult to perform but from a technical point of view the feasibility of this network by cascading a few compensated segments by considering that each segment is statistically independent (i.e. uncorrelated) from the others, thus the instability of the total link scales as the square root of the number of segments.

\begin{tabular}{|l|c|c|}
\hline & $86 \mathrm{~km}$ distribution system @ 1GHz & $186 \mathrm{~km}$ distribution system @ $1 \mathrm{GHz}$ \\
\hline Short-term ADEV & $3-510-15 @ 1 \mathrm{~s}$ & $1-3 \times 10-14 @ 1 \mathrm{~s}$ \\
\hline Long-term ADEV & $2-3 \times 10-18$ at 1 day & $\sim 10-17$ at 1 day \\
\hline
\end{tabular}

Table 1Summary of the major results

\section{Conclusions.}

We have demonstrated for the first time the long-distance transfer of both short-term and long-term frequency stability of frequency standards, with low phase noise via telecom optical fibres. A stability of about $1 \times 10^{-14}$ at one second and $10^{-17}$ for one day integration has been obtained on an optical link of 2x43 km at 100MHz.

By upgrading the systems to one $1 \mathrm{GHz}$ and adding the polarisation scrambler we have obtained the best frequency stability ever archived by an optical link. Over $86 \mathrm{~km}$ the optoelectronic compensator shows a frequency stability of a few $10^{-15}$ at one second and $2-3 \times 10^{-18}$ for one day integration times.

This study has triggered the interest in fiber frequency transfer over long distance among different laboratories in Europe. Moreover in the US and in Japan similar studied are under development.

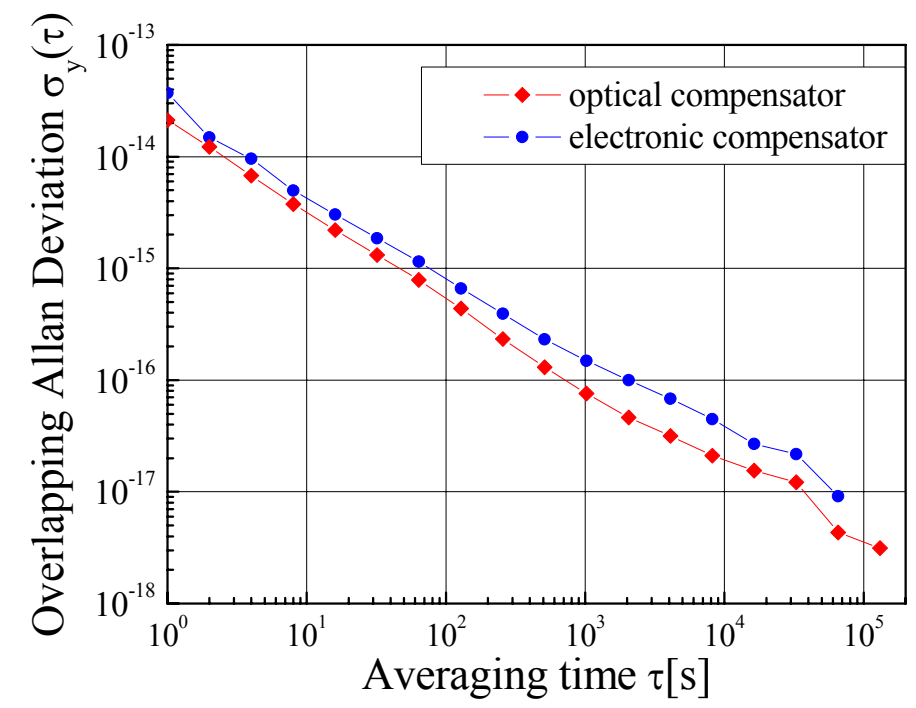

Figure 11: Preliminary relative frequency stability of a $186 \mathrm{~km}$ link. (triangles: electronic compensator ; squares : optical compensator). 


\section{Appendix: Summary Table of the results}

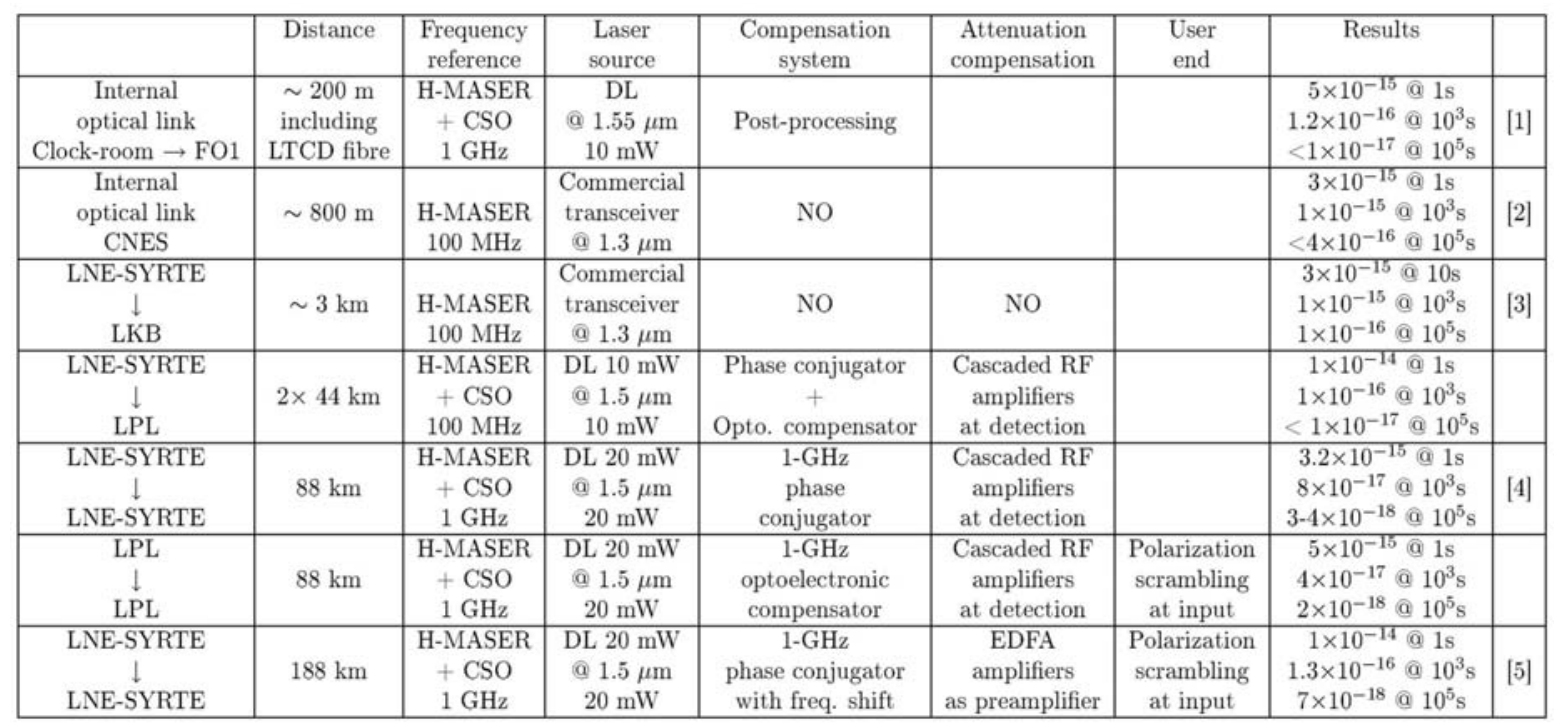

The different configurations for the conducted experiments are:

[1]: Experiments of LNE-SYRTE (atomic fountain, optical clocks, femtosecond laser, inertial sensors ...) are connected by LTCD optical fibres (between buildings) and standard SMF-28 fibre (inside laboratories), to the clock room facilities (H-MASERs and one CSO). From these frequency standards, reference signals at $100 \mathrm{MHz}$ and $1 \mathrm{GHz}$ are produced according to the applications. In the previous table, we present the link delivering reference signal to the Caesium atomic fountain FO1, needed for generation of the atomic interrogation signal, via an homemade frequency synthesizer. Due to the laboratory environment, the short-term frequency stability is not degraded and only thermal effects (air-conditioning and daily fluctuations) corrupt the medium and longterm frequency stability of the distribution. Consequently, two different links are used for a passive compensation based on a data post-computing by differential measurement.

[2]: The CNES optical link operates with standard optical fibre in closed environment. This distribution system allows for a connection between the PHARAO test facilities and the CNES Time and Frequency laboratory, where GPS stations and HMASER are installed. The goal of this link is both to deliver a reference signal for the characterization of PHARAO and to perform a distant comparison with the LNE-SYRTE atomic fountains by GPS. No active compensation is used since the requirements and the expected performance of PHARAO.

[3]: The LNE-SYRTE to LKB fibre link, using the France Telecom Paris network, is based on the distribution of a reference signal coming from one of the filtered LNE-SYRTE H-MASERs at $100 \mathrm{MHz}$.

[4]: The $88-\mathrm{km}$ optical link is performed by connecting the two $43-\mathrm{km}$ optical fibres of the LNE-SYRTE to LPL link together.

[5]: To extend the LNE-SYRTE to LPL optical link, we use some fibre spools, composed of SMF-28 fibre with an attenuation slightly below $0.2 \mathrm{~dB} / \mathrm{km}$ and a very low PMD which is of the order of $0.05 \mathrm{ps} / \mathrm{km}^{1 / 2}$. Fibre spools are more constrained to thermal effects due to ideal mechanical mounting. In contrast with the LNE-SYRTE to LPL link, perturbations are linearly distributed along the fibre since all the fibre spools are submitted to these perturbations.

\section{The Allan variance}

The Allan variance, is a measurement of stability in clocks and oscillators. It is also known as the two-sample variance. It is defined as one half of the time average of the squares of the differences between successive readings of the frequency deviation sampled over the sampling period. The Allan variance depends on the time period used between samples: therefore it is a function of the sample period, as well as the distribution being measured, and is displayed as a graph rather than a single number. A low Allan variance is a characteristic of a clock with good stability over the measured period. The Allan variance is given by

$$
\sigma_{y}^{2}(\tau)=\frac{1}{2}\left\langle\left(y_{n+1}-y_{n}\right)^{2}\right\rangle
$$


where $y_{n}$ is the normalized frequency departure, averaged over sample period $n$, and $\tau$ is the time per sample period. The samples are taken with no dead-time between them.

$$
y_{n}=\left\langle\frac{\delta v}{v}\right\rangle_{n}
$$

where $v$ is the frequency, $\delta v$ is the frequency error, and the average is taken over sampling period $n$. For a clock, the time error, $x_{n}$, at sampling period $n$, is the sum of the preceding frequency errors, given by

$$
x_{n}=x_{n+2}+\sum_{i=0}^{n-1} y_{i}
$$

This can be reversed to compute frequency error from time error measurements

$$
y_{n}=x_{n+1}-x_{n}
$$

which leads to the equation for Allan variance in terms of time errors:

$$
\sigma_{y}^{2}(\tau)=\frac{1}{2}\left\langle\left(x_{n+2}-2 x_{n+1}-x_{n}\right)^{2}\right\rangle
$$

Just as with standard deviation and variance, the Allan standard deviation (ADEV) is defined as the square root of the Allan variance. The practical realisation of the ADEV is the following:

$$
\sigma_{y}(\tau)=\sqrt{\frac{1}{2(M+1)} \sum_{n=1}^{M}\left(x_{n+2}-2 x_{n+1}-x_{n}\right)^{2}}
$$

where $\mathrm{M}$ is the number of samples.

\section{Acknowledgments}

This work was supported by the European Space Agency / ESOC. Thanks to Observatoire de Paris, the Laboratoire National de métrologie et d'Essais (LNE), Paris 13 University, the French Research Ministry, CNRS, and the Laboratoire d'Optronique, GIS FOTON, ENSSAT Lannion. The authors thank D. Chambon, Anne Amy-Klein, Christophe Daussy and Christian Chardonnet..

\section{References}

[1] P.Wolf, S. Bize, A. Clairon, A. N. Luiten, G. Santarelli and M. E. Tobar, Physics Review Letter, 90, 060402, 2003

[2] S. Bize et al., J. Phys. B, Atom. Mol. Opt. Phys. 38,pp. 449-468, 2005

[3] M. Fischer et al., Phys. Rev. Lett. 92, 230802, 2004

[4] E. Peik et al., Phys. Rev. Lett. 93, 170801, 2004

[5] J. Frisch, D. Bernstein, D. Brown and E. Cisneros, inIEEE Particle Accelerator Conference, 2002

[6] B. Shillue, ALMA Memo 443, 2002 (website:http://www.alma.nrao.edu/memos/)

[7] K.H. Sato et al, in IEEE Trans. Instrum. Meas., vol.41, pp. 385-389, 1992.

[8] R.T. Logan, G.F. Lutes, in Proc. of IEEE FrequencyControl Symposium, 1992, p. 310-316.

[9] M. Calhoun et al, in Proc. of the 32nd Annual Precise Time and Time Interval Systems and Applications Meeting, 2000, pp. 331-340.

[10] S. Bize et al, in C.R. Physique, vol. 5, 829, 2004

[11] I. Courtillot et al, in Physical Review A, vol. 68, pp. 030501, 2003

[12] F. Riehle et al, in Appl. Phys. B, vol. 648, pp. 229-244,2004

[13] U. Tanaka et al, in J. Phys. B, Atom. Mol. Opt. Phys. 36, pp. 545-551, 2003

[14] H. S. Margolis, G. P. Barwood, G. Huang, H. A. Klein, S. N. Lea, K. Szymaniec and P. Gill, in Science, vol.306, pp. 1355-1358, 2004

[15] Th. Udem et al, in Physical Review Letters, vol. 86, 2001

[16] L.S. Ma et al, in Science, vol. 303, pp. 1843, 2004

[17] A. Amy-Klein et al, in Appl. Phys. B, vol. 78, pp. 25-30, 2004

[18] A. Bauch et al, in Metrologia, vol. 43, pp. 109-120,2006 
[19] J. Ye et al, in J. Opt. Soc. Am. B, vol. 20, pp. 1459,2003

[20] K. W. Holman, D. J. Jones, D. D. Hudson and J. Ye,in Optics letters, vol. 29, 2004.

[21] K. Sato, T. Hara, S. Kuji, K. Asari, M. Nishio and N. Kawano, in IEEE Trans.on Instr. and Meas, vol. 49, pp. 19-24, 2000

[22] M. Calhoun, R. Sydnor and W. Diener, in The Interplanetary Network Progress Report, IPN PR 42-148, pp. 1-11, 2001.

[23] V. Bernard et al, in IEEE J. Quantum Electron, vol. 33, pp. 1282, 1997

[24] D. Chambon et al, in Rev.of Scient Inst., vol., 76, 094704, 2005

[25] C. Daussy et al, in PRL, vol. 94, 203904, 2005 and F. Narbonneau et al, Rev. of Scient. Instr., 77, 064701, 2006. 